\title{
LOOP GROUPS, ELLIPTIC SINGULARITIES AND PRINCIPAL BUNDLES OVER ELLIPTIC CURVES
}

\author{
STEFAN HELMKE \\ Research Institute for Mathematical Sciences \\ Kyoto University, Kyoto 606-8502, Japan \\ E-mail: helmke@kurims.kyoto-u.ac.jp \\ PETER SLODOWY
}

Mathematisches Seminar der Universität Hamburg

Universität Hamburg, D-20146 Hamburg, Germany

\begin{abstract}
There is a well known relation between simple algebraic groups and simple singularities, cf. [5], [28]. The simple singularities appear as the generic singularity in codimension two of the unipotent variety of simple algebraic groups. Furthermore, the semi-universal deformation and the simultaneous resolution of the singularity can be constructed in terms of the algebraic group. The aim of these notes is to extend this kind of relation to loop groups and simple elliptic singularities. It is the successful completion of work begun long ago, [29], cf. also [31], [30], [32] for more general situations.
\end{abstract}

1. Simple groups and simple singularities. (For details cf. [28].) Let $G$ be a simple and simply connected algebraic group over $\mathbb{C}$. Then, $G$ acts by conjugation on itself and the ring of invariant functions on $G$ is a polynomial ring in the fundamental characters of $G$

$$
\mathbb{C}[G]^{G}=\mathbb{C}\left[\chi_{1}, \ldots, \chi_{\ell}\right]
$$

The induced map

$$
G \longrightarrow \operatorname{Spec} \mathbb{C}\left[\chi_{1}, \ldots, \chi_{\ell}\right] \simeq \mathbb{C}^{\ell}
$$

2000 Mathematics Subject Classification: Primary: 14B07, 14L32. Secondary: 14H52, 14H60, $32 \mathrm{~S} 25$.

Key words and phrases: loop groups, elliptic singularities, deformations, principal bundles.

A previous version of these notes appeared first in [11] and [12]. The problems which where formulated in Sections 5 and 10 where settled during a stay of the second author at RIMS in spring 2002. After his sudden death a few months later, the first author updated the previous version, so that the current version contains now the solutions to those problems.

The paper is in final form and no version of it will be published elsewhere. 
is called the adjoint quotient map for $G$. The group $G$ also acts by the adjoint representation on its Lie algebra $\mathfrak{g}$ and the ring of invariant functions on $\mathfrak{g}$ is also a polynomial ring in $\ell=\operatorname{rank} G$ variables (in this case, for arbitrary $\mathfrak{g}$, the generators cannot so easily be described). The natural map

$$
\chi: \mathfrak{g} \longrightarrow \mathfrak{g} / / G \simeq \mathbb{C}^{\ell}
$$

is similarly called the adjoint quotient map for $\mathfrak{g}$. The zero fiber $N=\chi^{-1}(0)$ is called the nilpotent variety, since it consists of all the nilpotent elements of $\mathfrak{g}$. Under the action of $G$ the nilpotent variety decomposes into a finite number of orbits. In particular there is one open and dense orbit, the so called regular orbit. Moreover, there is a unique orbit of codimension two in $N$, the so called subregular orbit. Now let $T$ be a transversal slice to the subregular orbit $\left(T \simeq \mathbb{C}^{\ell+2}\right)$ and for simplicity assume that $G$ is of type $A D E$. Then, due to E. Brieskorn [5], the restriction of the adjoint quotient map $\chi$ to the transversal slice $T$

$$
\left.\chi\right|_{T}: \mathbb{C}^{\ell+2} \longrightarrow \mathbb{C}^{\ell}
$$

is the semi-universal deformation of the simple hypersurface singularity $T \cap N$, where the equation for $T \cap N$ is listed in Figure 1 .

\begin{tabular}{|c|r|}
\hline$G$ & \multicolumn{1}{|c|}{$T \cap N$} \\
\hline$A_{\ell}=S L_{\ell+1}$ & $x^{\ell+1}+y^{2}+z^{2}=0$ \\
$D_{\ell}=\operatorname{Spin}_{2 \ell}$ & $x^{\ell-1}+x y^{2}+z^{2}=0$ \\
$E_{6}$ & $x^{4}+y^{3}+z^{2}=0$ \\
$E_{7}$ & $x^{3} y+y^{3}+z^{2}=0$ \\
$E_{8}$ & $x^{5}+y^{3}+z^{2}=0$ \\
\hline
\end{tabular}

Figure 1. Simple Singularities

These singularities are characterized in many ways. For example they are the only 2-dimensional hypersurface singularities which can be deformed into only a finite number of other singularities (up to isomorphism). For that reason they were called simple singularities, cf. [1]. If $G$ is of type $B C F G$, the singularity $T \cap N$ carries an extra finite symmetry and $\left.\chi\right|_{T}$ is only the invariant part of the semi-universal deformation of $T \cap N$.

The restriction of the adjoint quotient map for $G$ to a transversal slice at a subregular unipotent orbit is also a semi-universal deformation. But there is an important technical difference. The map $\left.\chi\right|_{T}$ is quasi-homogeneous with respect to natural $\mathbb{C}^{*}$-actions on $T$ and $\mathbb{C}^{\ell}$ with only positive weights. Such $\mathbb{C}^{*}$-actions do not exist for $G$.

2. Simple elliptic singularities. Let $E$ be an elliptic curve and $L \longrightarrow E$ be a holomorphic line bundle over $E$ of degree $d<0$. Then one can contract the zero section of $L$ to a surface singularity $X$. It turns out that $X$ is a complete intersection, if and only if $d \geqslant-4$. For $d=-4$, the singularity $X$ is the intersection of two quadrics in $\mathbb{C}^{4}$. More precisely, $X$ is the cone over the elliptic curve $E$ embedded by the line bundle $L^{*}$ of degree 4 in $\mathbb{P}^{3}$. This singularity is called of type $\tilde{D}_{5}$.

For $d \geqslant-3$, the singularity $X$ is a hypersurface singularity of type $\tilde{E}_{9+d}$. Its equation is listed in Figure 2. 
Here, $\lambda$ is a complex parameter which depends on the elliptic curve $E$. These singularities and their deformations have been thoroughly studied by K. Saito [23], [24], [25], [26], E. J. N. Looijenga [16], [17], [18], H. C. Pinkham [20], and J. Y. Mérindol [19]. It turns out that they deform only into elliptic singularities of the same type with different $\lambda$ and into simple singularities. For that and other reasons they are called simple elliptic singularities.

\begin{tabular}{|c|c|c|}
\hline$\tilde{E}_{8}$ & $d=-1$ & $x^{6}+y^{3}+z^{2}+\lambda x y z=0$ \\
$\tilde{E}_{7}$ & $d=-2$ & $x^{4}+y^{4}+z^{2}+\lambda x y z=0$ \\
$\tilde{E}_{6}$ & $d=-3$ & $x^{3}+y^{3}+z^{3}+\lambda x y z=0$ \\
\hline
\end{tabular}

Figure 2. Simple elliptic singularities

3. Loop groups. The (holomorphic) loop group of the simple algebraic group $G$ is defined by

$$
\mathcal{L} G=\left\{\varphi: \mathbb{C}^{*} \longrightarrow G \mid \varphi \text { is holomorphic }\right\} .
$$

The infinite dimensional group $\mathcal{L} G$ has a universal central extension

$$
1 \longrightarrow \mathbb{C}^{*} \longrightarrow \tilde{\mathcal{L}} G \longrightarrow \mathcal{L} G \longrightarrow 1
$$

which can be defined via the embedding of $\mathcal{L} G$ into the "differentiable" loop group studied by Pressley and Segal [21].

Now, $\mathbb{C}^{*}$ acts on $\mathcal{L} G$ by the formula $(q \varphi)(z)=\varphi(q z)$ and this action can be lifted to $\tilde{\mathcal{L}} G$. The semi-direct product of $\tilde{\mathcal{L}} G$ with $\mathbb{C}^{*}$ is denoted by

$$
\hat{\mathcal{L}} G=\tilde{\mathcal{L}} G \rtimes \mathbb{C}^{*} .
$$

It may be viewed as a certain completion of some affine Kac-Moody group (corresponding to polynomial loops, cf. e.g. [15], [14]). Such a group has $\ell+1$ fundamental highest weight representations. The corresponding characters are convergent for $(\tilde{\varphi}, q)$ with $q \in D^{*}$, where $D^{*}=\{q \in \mathbb{C}|0<| q \mid<1\}$, cf. [6] for the polynomial case. Hence, there is a map

$$
\begin{aligned}
& \hat{\mathcal{L}} G \\
& \tilde{\mathcal{L}} G \times D^{*} \stackrel{\hat{\chi}}{\longrightarrow} \mathbb{C}^{\ell+1} \times D^{*},
\end{aligned}
$$

where $\hat{\chi}$ is given by the $\ell+1$ fundamental characters and the second projection. This map is called the adjoint quotient map for $\hat{\mathcal{L}} G$ (cf. [29], [31], [30], [32] in the case of "formal" loop groups; it has some good categorical properties only after a certain partial completion, [33]).

4. Subregular singularities. In the sequel we will fix a number $q \in D^{*}$. The zero fiber $U_{q}=\hat{\chi}^{-1}(0, q)$ is called the unstable variety for reasons we will see later. In this section we are going to describe the singularities of the unstable variety in codimension two for all $\hat{\mathcal{L}} G$, where $G$ is of type $A D E$. For this purpose we will call an $\hat{\mathcal{L}} G$ orbit in $U_{q}$ regular, if it has codimension zero in $U_{q}$ and it is called subregular if it has codimension two in $U_{q}$. Actually all orbits in $U_{q}$ have finite codimension and there is never one of codimension one. 
4.1. Case $A_{1}$. There are one regular and one subregular orbit in $U_{q}$. Let $S$ be the subregular orbit and $T_{S}$ a transversal slice to $S$. Then the singularity of $U_{q} \cap T_{S}$ is of type $\tilde{D}_{5}$.

4.2. Case $A_{\ell}, \ell>1$. There are $\ell$ regular orbits $R_{1}, \ldots, R_{\ell}$ and each intersection $\bar{R}_{i} \cap \bar{R}_{i+1}$ contains a one-parameter family of subregular orbits. For every subregular orbit, the singularity of $U_{q} \cap T_{S}$ is of type $A_{\infty}$ i.e. two smooth, transversally crossing surfaces:

$$
\left\{(x, y, z) \in \mathbb{C}^{3} \mid y^{2}+z^{2}=0\right\} .
$$

4.3. Case $D_{\ell}, \ell>5$. There are one regular orbit and a one-parameter family of subregular orbits. Generically the singularity of $U_{q} \cap T_{S}$ is of type $A_{\infty}$, but for four different subregular orbits it is of type $D_{\infty}$ (cf. Fig. 3):

$$
\left\{(x, y, z) \in \mathbb{C}^{3} \mid x y^{2}+z^{2}=0\right\} .
$$

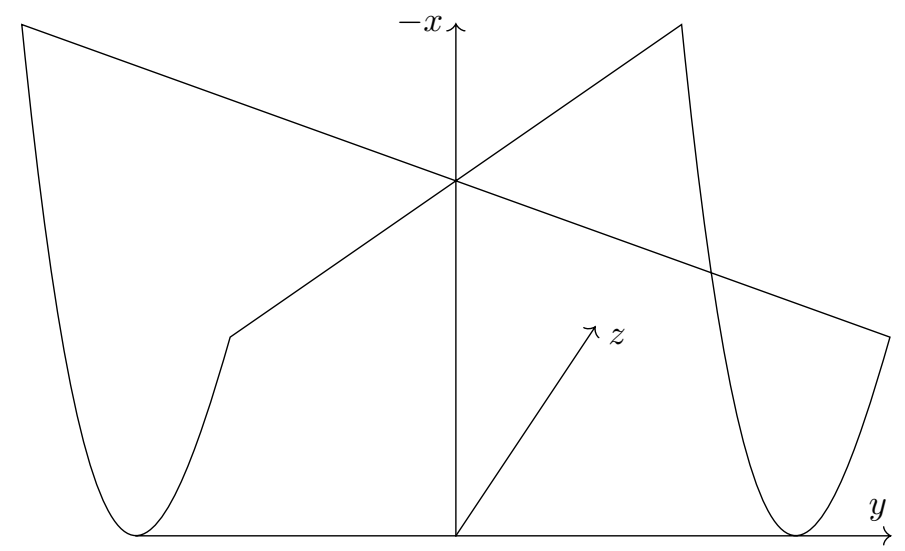

Figure 3. The Whitney umbrella $D_{\infty}$

4.4. Case $D_{4}$. The same as for $D_{\ell}$ with $\ell>5$, but there are three such one-parameter families of subregular orbits.

4.5. Case $D_{5}$. Besides the one-parameter family of subregular orbits there are two more subregular orbits. The singularity of $U_{q} \cap T_{S}$ for those two orbits is of type $\tilde{D}_{5}$.

4.6. Case $E_{6}$. There are one regular and two subregular orbits. The singularity of $U_{q} \cap T_{S}$ is of type $\tilde{E}_{6}$.

4.7. Case $E_{7}$. There are one regular and one subregular orbit. The singularity of $U_{q} \cap T_{S}$ is of type $\tilde{E}_{7}$.

4.8. Case $E_{8}$. There are one regular and one subregular orbit. The singularity of $U_{q} \cap T_{S}$ is of type $\tilde{E}_{8}$. 
5. Deformations. The restriction of the adjoint quotient map $\hat{\chi}$ to a transversal slice $T_{S}$ to a subregular orbit $S$ is a deformation of the singularity $U_{q} \cap T_{S}$. In the cases $\tilde{E}_{\ell}$ and also for the two special subregular orbits of $\tilde{D}_{5}$, this turns out to be the semiuniversal deformation. But in the other cases, the singularity $U_{q} \cap T_{S}$ is non-isolated and the base of its semi-universal deformation is infinite dimensional. But as in the case of an algebraic group of type $B C F G$ there is an extra symmetry and the restriction of the adjoint quotient map is the invariant part of the semi-universal deformation. More precisely, in a loop group of type $\tilde{A}_{\ell}$ or $\tilde{D}_{\ell}$ one can find a particular unstable orbit $S^{\prime}$ and a transversal slice $T_{S^{\prime}}$ to this orbit, such that every subregular orbit (of a given family in case $\tilde{A}_{\ell}$ ) has a non-trivial intersection with $T_{S^{\prime}}$. The stabilizer of $S^{\prime}$ contains two $\mathbb{C}^{*}$-actions and the positive weight space $T^{+}$of the first one is a subspace of $T_{S^{\prime}}$, on which the second $\mathbb{C}^{*}$ still acts. Denote by $T_{0}^{+} \subset T^{+}$the open subset of semi-stable points with respect to this $\mathbb{C}^{*}$-action, i.e. the union of those $\mathbb{C}^{*}$-orbits, whose closure does not contain 0 . In general, the subgroup $H$ of the loop group which stabilizes $T^{+}$ is an extension of $\mathbb{C}^{*}$ by an unipotent group and the orbit space $T_{0}^{+} / H$ always exists. The restriction of the adjoint quotient map to $T^{+}$induces a deformation of the surface singularity $Z=T_{0}^{+} \cap U_{q} / H$. In addition, there is another one-parameter deformation of this singularity with weight zero, which comes from the choice of $S^{\prime}$ and these two deformations together form the semi-universal deformation of $Z$. In each case, one can also construct the singularity $Z$ as a hypersurface of a smooth 3-dimensional manifold $X$. In the following, we will shortly describe this 3-dimensional manifold and the embedding $Z \subset X$.

5.1. If the loop group is of type $A_{\ell}$, then $X$ is the total space of the sum of two line bundles over an elliptic curve $E$. The degrees of those line bundles depend on $\ell$ and also on the subregular family. The singularity $Z$ is the union of two smooth hypersurfaces corresponding to the two regular orbits of the loop group, whose closure contain the chosen subregular family, intersecting transversally in the elliptic curve $E$.

5.2. If the loop group is of type $D_{\ell}$, then $X$ is the total space of the sum of two line bundles over a smooth rational curve $\mathbb{P}^{1}$. The degree of those line bundles is $\frac{1}{2}(\ell-6)$ if $\ell$ is even. If $\ell$ is odd, one line bundle has degree $\frac{1}{2}(\ell-5)$ and the other one has degree $\frac{1}{2}(\ell-7)$. The singularity $Z$ is an irreducible hypersurface in $X$ whose normalization is smooth. Its singular locus is the rational curve $\mathbb{P}^{1} \subset X$ where $Z$ intersects itself transversally except for four points. At those four points, $Z$ has a singularity of type $D_{\infty}$. In this case, the elliptic curve appears as a double cover of the rational curve $\mathbb{P}^{1}$, which is branched exactly over those four exceptional points.

6. Principal bundles over elliptic curves. We start with an observation due to E. J. N. Looijenga (unpublished, however, see e.g. [4]). Let $P \longrightarrow E$ be a principal $G$-bundle over the elliptic curve $E=\mathbb{C}^{*} / q^{\mathbb{Z}}$ and let $\pi: \mathbb{C}^{*} \longrightarrow E$ be the natural projection. Then, the pull back $\pi^{*} P$ of $P$ to $\mathbb{C}^{*}$ is holomorphically trivial since $G$ is connected. Therefore one has $P \simeq\left(\mathbb{C}^{*} \times G\right) / \mathbb{Z}$, where the generator $1 \in \mathbb{Z}$ acts by

$$
(z, g) \longmapsto(q z, \varphi(z) \cdot g)
$$


and $\varphi \in \mathcal{L} G$. We shall call $\varphi$ a multiplier for $P$. Mapping, in the other direction, a pair $(\varphi, q)$ to the corresponding principal bundle $P \simeq\left(\mathbb{C}^{*} \times G\right) / \mathbb{Z}$ one obtains a surjective map

$$
\begin{aligned}
& \mathcal{L} G \rtimes \mathbb{C}^{*} \\
& \cup \\
& \mathcal{L} G \times q \longrightarrow\{\text { holomorphic } G \text {-bundles over } E \text { \} } .
\end{aligned}
$$

As is easily checked, this map induces a bijection from the set of $\mathcal{L} G$-conjugacy classes in $\mathcal{L} G \times q$ to the isomorphism classes of holomorphic $G$-bundles on $E$ :

$$
(\mathcal{L} G \times q) / \mathcal{L} G \stackrel{1-1}{\longrightarrow}\{\text { isomorphism classes of holomorphic } G \text {-bundles over } E\} .
$$

Adding the "semi-direct" $\mathbb{C}^{*}$-action on the left side corresponds to allowing translations of the base $E$ on the right side.

According to V. Baranovsky and V. Ginzburg [4], an element of $\tilde{\mathcal{L}} G \times q$ belongs to the variety $U_{q}$ if and only if the corresponding $G$-bundle over $E$ is unstable (in the sense of [9], [22]). For that reason, we call $U_{q}$ the unstable variety.

From this fact one can deduce that there are no nontrivial continuous $\mathcal{L} G$-invariant functions on $\mathcal{L} G \times q$. This is the geometric reason why, for our purpose, we need the central extension $\hat{\mathcal{L}} G$. Inside the centrally extended $\tilde{\mathcal{L}} G \times q$ each semi-stable orbit contains only finitely many orbits in its closure and there is exactly one closed orbit in each such closure (cf. [29] for the case of "formal" loops). Note that centralizers may not be preserved under central extensions. This may happen to elements in $U_{q}$, but it does not occur for semistable ones.

7. Principal bundles and Levi subgroups. The structure group of a $G$-bundle over a curve can, by general reasons (e.g. [2]), always be reduced to a Borel subgroup of $G$. But if the bundle is unstable and the curve is elliptic, it can be further reduced. In fact, in the case of vector bundles, the instability means that there is some subbundle of positive degree and on an elliptic curve this has to be a direct summand. The general case can be reduced to that special case, cf. [9]. Therefore the structure group reduces to a Borel subgroup of some Levi subgroup of $G$ (i.e. the centralizer $L=C_{G}(H)^{\circ}$ of some torus $H \subset G)$.

In the other direction, one may construct "minimally" unstable $G$-bundles by extending to $G$ the structure group of a suitable $B(L)$-bundle where $B(L)$ is a Borel subgroup of some Levi subgroup $L$ of $G$. If $L$ is maximal, i.e. $Z(L)^{\circ} \simeq \mathbb{C}^{*}$, and the semi-simple components of $L$ are of type $A_{n}$ this construction often leads to an essentially unique (up to translation in the base) unstable $G$-bundle. But in some cases $\left(D_{n}\right)$ or if $Z(L)^{\circ} \simeq\left(\mathbb{C}^{*}\right)^{2}$, one gets an essential one-parameter family of unstable $G$-bundles. This has been extensively investigated in [13]. In the following, we will give a short summary of some our results.

For example, if $G$ is of type $E_{\ell}$, the Dynkin diagrams of the Levi subgroups corresponding to the regular and subregular orbits are the following (the regular cases correspond 
to those of the construction in Friedman-Morgan-Witten [10, Section 5]):

$\left(E_{\ell}\right)$
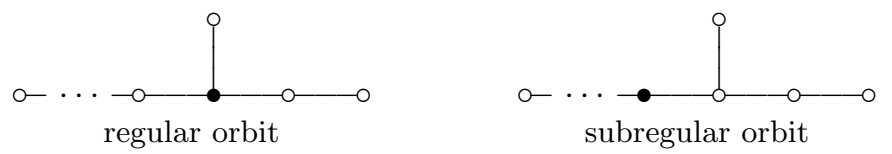

Here $L=C_{G}(H)^{\circ}$, where $H$ is the subtorus of the maximal torus spanned by the one-parameter subgroup corresponding to the filled vertex (i.e. proportional to the corresponding fundamental co-weight). In the case $E_{6}$ there is a symmetry of the diagram which leads to two subregular orbits.

In the case $D_{\ell}$ the Dynkin diagrams of regular and subregular orbits are
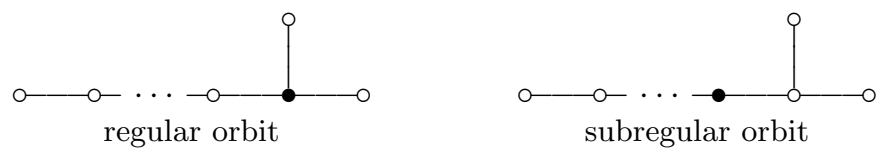

There is, in fact, a whole one-parameter family of subregular orbits. In the case $D_{4}$, one gets three such families by the symmetry of the diagram. In the case $D_{5}$ there are the following two more subregular elements
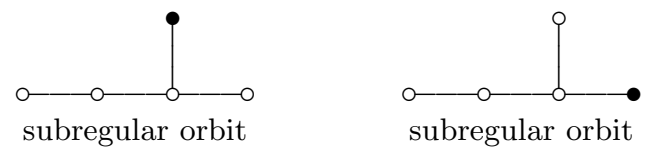

Finally, in the case $A_{\ell}$ with $\ell>1$ we have

regular orbit

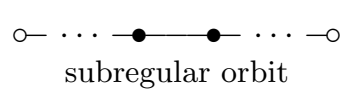

The case $A_{1}$ is a little special. Every unstable rank two vector bundle with trivial determinant is of the form $L \oplus L^{*}$ for some line bundle $L$ of degree $d>0$. The regular element corresponds to $d=1$ and the subregular to $d=2$.

8. The case $\operatorname{Spin}_{10}$. There is one subregular $\operatorname{Spin}_{10}$-bundle which corresponds to the Levi subgroup $\mathbb{C}^{*} \cdot S L_{5} \subset \operatorname{Spin}_{10}$. This is very easy to describe. There is a unique indecomposable rank 5 vector bundle $V$ with $\operatorname{det} V=L^{2}$, where $L$ is a line bundle of degree 1 . The bundle

$$
V \oplus V^{*}
$$

is a $S O_{10}$-bundle with trivial second Stiefel Whitney class. Hence, the structure group can be reduced to $\operatorname{Spin}_{10}$ and this is the subregular $\operatorname{Spin}_{10}$-bundle we are looking for. The deformations of this bundle are given by extensions

$$
0 \longrightarrow V^{*} \longrightarrow V_{\xi} \longrightarrow V \longrightarrow 0
$$

where the extension class $\xi \in \operatorname{Ext}^{1}\left(V, V^{*}\right)=H^{1}\left(V^{*} \otimes V^{*}\right)$ belongs to the subgroup $H^{1}\left(\bigwedge^{2} V^{*}\right)$. As a $\operatorname{Spin}_{10}$-bundle, $V \oplus V^{*}$ has one more deformation, namely the oneparameter deformation of $L$. But the group $\hat{\mathcal{L}} G$ contains the translations of the elliptic curve and hence we may ignore those deformations. The transversal slice to the orbit 
corresponding to $V \oplus V^{*}$ is therefore

$$
T_{S} \simeq H^{1}\left(\bigwedge^{2} V^{*}\right) \times D^{*}
$$

We want to see that $U_{q} \cap T_{S}$ has a $\tilde{D}_{5}$ singularity. For this purpose we have to find all $\xi$ for which $V_{\xi}$ is unstable. By definition $V_{\xi}$ is unstable if and only if there exists an indecomposable proper subbundle $W \subset V_{\xi}$ of positive degree. Since then one has

$$
\operatorname{Hom}(W, V) \neq 0
$$

it follows that the degree of $W$ is 1 and its rank has to be 3,4 or 5 . Actually 4 and 5 can be excluded from the fact that $V_{\xi}$ is a $\operatorname{Spin}_{10}$-bundle. Now, for every indecomposable rank 3 bundle $W$ of degree 1 one has $\operatorname{Hom}(W, V) \simeq \mathbb{C}$ and each nontrivial morphism $W \longrightarrow V$ is injective. From this we get the following commutative diagram

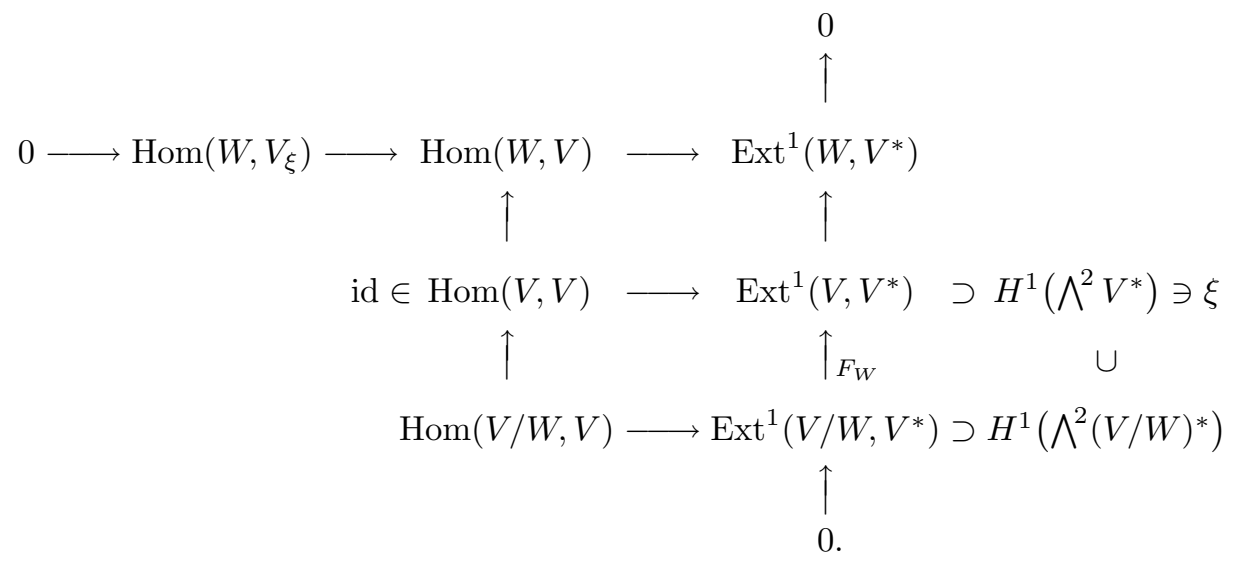

It shows that $V_{\xi}$ is unstable if and only if

$$
\xi \in \operatorname{Im} F_{W} \cap H^{1}\left(\bigwedge^{2} V^{*}\right)=H^{1}\left(\bigwedge^{2}(V / W)^{*}\right) \simeq \mathbb{C}
$$

for some indecomposable vector bundle $W$ of rank 3 and degree 1.

The vector bundle $\bigwedge^{2} V^{*}$ has rank 10 and degree -8 . It is actually the direct sum of a vector bundle $\tilde{V}$ of rank 5 and degree -4 with itself. Moreover, one can show that there is a canonically defined subbundle $\tilde{V} \subset \wedge^{2} V^{*}$ with the following property. For every choice of $W$, the line $\operatorname{Im} F_{W} \cap H^{1}\left(\bigwedge^{2} V^{*}\right)$ is contained in the four dimensional subspace $H^{1}(\tilde{V}) \subset H^{1}\left(\bigwedge^{2} V^{*}\right)$.

Recall, that due to M. F. Atiyah's work [2] the map $W \longmapsto \operatorname{det} W$ is a bijection between indecomposable rank 3 vector bundles of degree 1 and $\operatorname{Pic}^{1}(E) \simeq E$. Therefore, the unstable locus in $H^{1}\left(\bigwedge^{2} V^{*}\right)$ is the cone over the image of the natural morphism

$$
\begin{aligned}
\Phi: E & \longrightarrow \mathbb{P}\left(H^{1}(\tilde{V})\right), \\
P & \longmapsto \operatorname{Im} F_{W_{P}} \cap H^{1}\left(\bigwedge^{2} V^{*}\right),
\end{aligned}
$$

where $W_{P}$ is the rank 3 vector bundle with $\operatorname{det} W_{P}=\mathcal{O}(P)$. Finally one can identify the map $\Phi$ with the natural embedding of $E$ in $\mathbb{P}^{3}$ given by the line bundle $L^{4}$. Hence, the singularity $U_{q} \cap T_{S}$ is of type $\tilde{D}_{5}$.

Now, a simple argument using the $\mathbb{C}^{*}$-action on $T_{S}$ shows that the restriction of $\hat{\chi}$ to $T_{S}$ is in fact the semi-universal deformation of the zero fiber $U_{q} \cap T_{S}$. 
Similar but much simpler arguments can be used to settle the case of type $A_{1}$ where one also finds a $\tilde{D}_{5}$-singularity. The corresponding deformation is a subdeformation of the semi-universal one, preserving a certain symmetry group.

9. Transversal slices. In the cases $E_{6}, E_{7}$ and $E_{8}$ it is much harder to see the singularity directly. Instead, we proceed here in a way very similar to Brieskorn's original proof in [5] (cf. also [28]): Starting from the Jacobson-Morozov embedding of nilpotent elements into $\mathrm{sl}_{2}$-subalgebras of $\mathfrak{g}$ one can construct a special transversal slice $T_{S}$ admitting a $\mathbb{C}^{*}$-action such that the restricted adjoint quotient

$$
\chi: T_{S} \longrightarrow \mathfrak{g} / / G \simeq \mathbb{C}^{\ell}
$$

becomes $\mathbb{C}^{*}$-equivariant (with respect to the natural action on the base induced by the scalar action on $\mathfrak{g}$ ). Invoking a basic geometric property of this map (e.g. regularity of $\chi$ in all points of the special fiber $N \cap T_{S}$, except the origin; B. Kostant) the detailed knowledge of the weights and degrees of $\chi$ permits an identification of that fiber (a simple singularity) and, finally also, of its deformation (the semi-universal one).

In our situation, it turns out that all interesting unstable bundles have a nice description in terms of suitable multipliers which are obtained as follows. Take any element of infinite order in the affine Weyl group $\tilde{W}$ and lift it to an element $\tilde{\varphi} \in \tilde{\mathcal{L}} G$ (e.g. inside the normalizer of a maximal torus). Then $x=(\tilde{\varphi}, q) \in \hat{\mathcal{L}} G$ will represent an unstable bundle $P(x)$ over $E=\mathbb{C}^{*} / q^{\mathbb{Z}}$. For example, to get a regular unstable bundle one has to start from an affine Coxeter element (this is the "multiplier background" behind the construction in [10, Section 5]).

Using the Killing form and the Chevalley involution on the Lie algebra of $\hat{\mathcal{L}} G$ (cf. e.g. [15]) one can associate to $x$ an adjoint $x^{*}$ whose shifted connected centralizer $x . Z\left(x^{*}\right)^{o}$, where

$$
Z(h):=\{g \in \hat{\mathcal{L}} G \mid g . h=h . g\} \quad \text { for } h \in \hat{\mathcal{L}} G,
$$

is transversal to the orbit of $x$ inside $\tilde{\mathcal{L}} G \times q$. Moreover, exploiting the central extension and a "destabilizing" one-parameter subgroup of $G$ for $P(x)$ one can construct a $\mathbb{C}^{*}$-action on the manifold $T_{x}=x . Z\left(x^{*}\right)^{o}$ such that the restricted adjoint quotient

$$
T_{x} \stackrel{\hat{\chi}}{\longrightarrow} \mathbb{C}^{\ell+1} \times q,
$$

becomes $\mathbb{C}^{*}$-equivariant. Here the action on the base is given by the natural action of the centre $\mathbb{C}^{*}$ of $\hat{\mathcal{L}} G$ on the fundamental characters. If $x$ is subregular, the connected centralizer $Z\left(x^{*}\right)^{o}$ is unipotent (in the $E$-cases, at least) and it is possible to compute the weights of the above action. Invoking again basic geometric properties of $\hat{\chi}$ (i.e. regularity of $\hat{\chi}$ at regular elements, cf. G. Brüchert [6], and the classification of orbits (i.e. bundles) met by $T_{x}$ ) the explicit knowledge of the weights and degrees of $\hat{\chi}$ allows once more an identification of the singularity of $U_{q} \cap T_{x}$ (simple elliptic of the same type as $\hat{\mathcal{L}} G$ ) and its deformation by $\hat{\chi}$ (the semi-universal one of "negative weight"). A partial result in that last direction had already been obtained in [29], in the framework of "formal" loops.

Note that there is much freedom in the choice of transversal slices, the above one being in close analogy to the classical case. It is also possible to choose transversal slices 
inside the "polynomial" subgroup of $\hat{\mathcal{L}} G$. For the regular unstable orbit one may take an analogue of Steinberg's cross section, cf. [6].

10. Double loop algebras. In contrast to the finite dimensional case, the Lie algebra of $\hat{\mathcal{L}} G$ is not useful to construct simple elliptic singularities. This follows from work due to I. Frenkel and G. Segal, (cf. [8], [21], [27]), which relates the (co-)adjoint orbit structure of the Lie algebra of $\hat{\mathcal{L}} G$ with that of the finite dimensional group $G$. Instead of this, one has to consider now double loop algebras. These are defined by

$$
\mathcal{E} \mathfrak{g}=\left\{\varphi: S^{1} \times S^{1} \longrightarrow \mathfrak{g} \mid \varphi \text { is } \mathcal{C}^{\infty}\right\}
$$

There are two derivations $\partial / \partial \alpha$ and $\partial / \partial \beta$ acting on $\mathcal{E} \mathfrak{g}$, where a point in $S^{1} \times S^{1}$ is parametrized by $\left(e^{i \alpha}, e^{i \beta}\right)$. We are interested in the conjugacy classes of the semi-direct product

$$
\mathcal{E} \mathfrak{g} \rtimes\left(\mathbb{C} \frac{\partial}{\partial \alpha} \oplus \mathbb{C} \frac{\partial}{\partial \beta}\right) .
$$

Let us fix a derivation

$$
\bar{\partial}=\omega \frac{\partial}{\partial \alpha}+\eta \frac{\partial}{\partial \beta} \quad \text { with } \quad \operatorname{Im} \frac{\omega}{\eta}>0 .
$$

Then $\bar{\partial}$ defines a holomorphic structure on $S^{1} \times S^{1}$ by

$$
\begin{aligned}
& S^{1} \times S^{1} \\
& \cup \text { open } \\
& f: U \longrightarrow \mathbb{C} \quad \text { is holomorphic }: \Longleftrightarrow \bar{\partial} f=0 .
\end{aligned}
$$

With this holomorphic structure $S^{1} \times S^{1}$ becomes an elliptic curve $E$. Moreover, an element $\varphi \in \mathcal{E} \mathfrak{g}$ defines a holomorphic structure on the topologically trivial Ad $G$-bundle $E \times \mathfrak{g} \longrightarrow E$ by

$$
\begin{aligned}
& E \\
& \cup \text { open } \\
s: & U \longrightarrow \mathfrak{g} \text { is holomorphic }: \Longleftrightarrow(\bar{\partial}+\varphi) s=0,
\end{aligned}
$$

where $\varphi s$ is defined by matrix multiplication. The adjoint group $\mathcal{E} G$ corresponding to $\mathcal{E} \mathfrak{g}$ acts on $\mathcal{E} \mathfrak{g} \times \bar{\partial}$ as the gauge group of $E \times \mathfrak{g} \longrightarrow E$ and hence we have a bijection

$$
(\mathcal{E} \mathfrak{g} \times \bar{\partial}) / \mathcal{E} G \stackrel{1-1}{\longrightarrow}\{\text { isomorphism classes of holomorphic } G \text {-bundles over } E\}
$$

(cf. also [3], [7], [9]). This suggests that the simple elliptic singularities and their deformations should also appear in the double loop algebras $\mathcal{E} \mathfrak{g}$ (suitably enlarged by derivations and central extension). Indeed, the geometry of the loop group can be transferred to the double loop algebra via the following construction. Let

$$
\mathfrak{X}=\left\{\Phi: \mathbb{C}^{*} \longrightarrow G \mid \Phi \text { is } \mathcal{C}^{\infty} \text { and } \Phi_{q} \Phi^{-1} \text { is holomorphic }\right\},
$$

where $\Phi_{q}$ denotes the shifted function $\Phi_{q}(z)=\Phi(q z)$. The condition that $\Phi_{q} \Phi^{-1}$ is holomorphic, is actually equivalent to the condition that the smooth function $\Phi^{-1} \bar{\partial} \Phi$ is 
$q$-periodic, i.e. $\Phi_{q}^{-1} \bar{\partial} \Phi_{q}=\Phi^{-1} \bar{\partial} \Phi$. Hence, we get two maps

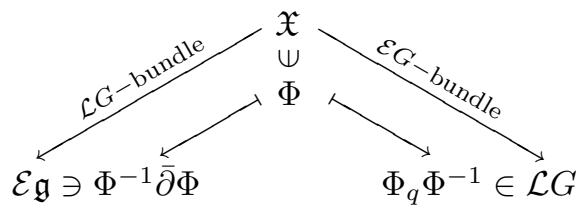

if we identify $\mathbb{C}^{*} / q^{\mathbb{Z}}$ with $S^{1} \times S^{1}$. The group $\mathcal{L} G$ acts by multiplication from the left and the group $\mathcal{E} G$ acts by multiplication from the right on $\mathfrak{X}$. With these actions, the map onto $\mathcal{E} \mathfrak{g}$ becomes a principal $\mathcal{L} G$-bundle and the map onto $\mathcal{L} G$ becomes a principal $\mathcal{E} G$ bundle as indicated in the diagram. The induced action of $\mathcal{E} G$ on $\mathcal{E} \mathfrak{g}$ is just the adjoint action on the coset $\mathcal{E} \mathfrak{g} \times \bar{\partial}$ and the induced action of $\mathcal{L} G$ on itself is the adjoint action on $\mathcal{L} G \times q$. This diagram can be considered as the analog of Frenkel's construction [8], which induces a one-to-one correspondence between adjoint orbits of the affine Lie algebra $\hat{\mathcal{L}} \mathfrak{g}$ (with fixed non-zero derivation) and conjugacy classes in $G$. To get invariant functions, we still have to add central extensions. Remember that the group $\tilde{\mathcal{L}} G$ is a $\mathbb{C}^{*}$-bundle over the loop group $\mathcal{L} G$. Denote by $\tilde{\mathfrak{X}}$ the pull-back of this $\mathbb{C}^{*}$-bundle to $\mathfrak{X}$ and let $\tilde{\mathcal{E}}=\tilde{\mathfrak{X}} / \mathcal{L} G$. Then, we get the commutative diagram

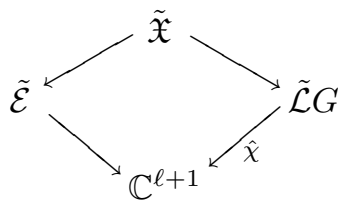

In particular, the fundamental characters of $\tilde{\mathcal{L}} G$ induce invariant functions on $\tilde{\mathcal{E}}$. It remains to identify the $\mathbb{C}^{*}$-bundle $\tilde{\mathcal{E}}$ over the double loop algebra $\mathcal{E} \mathfrak{g}$. It appears already in $[26]$. One has to take the semi-direct product $\hat{\mathcal{E}} \mathfrak{g}$ of the universal central extension

$$
0 \longrightarrow Z \longrightarrow \tilde{\mathcal{E}} \mathfrak{g} \longrightarrow \mathcal{E} \mathfrak{g} \longrightarrow 0
$$

of the double loop algebra $\mathcal{E} \mathfrak{g}$ with the two dimensional space of derivations as above. On this Lie algebra one has a natural invariant Killing form, whose radical $Z^{0}$ is a two codimensional subspace of the infinite dimensional $Z$. The quadratic form $Q$ associated to the Killing form is an invariant function on $\hat{\mathcal{E}} \mathfrak{g}$. Moreover, the Weyl group of $\hat{\mathcal{E}} \mathfrak{g}$ is the semi-direct product of the Weyl group of $\mathfrak{g}$ with a Heisenberg group. The centre $C \simeq \mathbb{Z}$ of this Heisenberg group lies in $Z$ and therefore, $\mathcal{E} G$ still acts on the quotient of $\hat{\mathcal{E}} \mathfrak{g}$ by $C$. Now, the line bundle $\tilde{\mathcal{E}}$ can be identified with the level set on $Q$ divided by this centre. More precisely,

$$
\tilde{\mathcal{E}} \simeq\left\{\varphi \in \tilde{\mathcal{E}} \mathfrak{g} / Z^{0} \times \bar{\partial} \mid Q(\varphi)=c\right\} / C \text { for any } c \in \mathbb{C} .
$$

This can be proved by reduction to the Cartan subalgebra. In particular, it follows from our construction that the 'holomorphic invariant functions' on $\hat{\mathcal{E}} \mathfrak{g}$ are generated by the two projections onto the space of derivations, the quadratic form $Q$ associated to the Killing form and the $\ell+1$ functions induced by the fundamental characters $\hat{\chi}$ of the Kac-Moody group $\hat{\mathcal{L}} G$.

The use of the Lie algebra $\hat{\mathcal{E}} \mathfrak{g}$ instead of the Lie group $\hat{\mathcal{L}} G$ has basically one advantage. The Killing form on the quotient algebra $\hat{\mathcal{E}} \mathfrak{g} / Z^{0}$ induces a symplectic form on adjoint 
orbits, the Kostant-Kirillov form. The restriction of this form to a transversal slice to a subregular orbit should be the 'primitive form' [24] as in the finite dimensional case [34]. But a proof of this is still unknown.

\section{References}

[1] V. I. Arnol'd, Normal forms for functions near degenerate critical points, the Weyl groups $A_{k}, D_{k}, E_{k}$, and Lagrangian singularities, Funktsional. Anal. i Prilozhen. 6:4 (1972), 3-25; English transl.: Funct. Anal. Appl. 6 (1972), 254-272.

[2] M. F. Atiyah, Vector bundles over an elliptic curve, Proc. London Math. Soc. (3) 7 (1957), 414-452.

[3] M. F. Atiyah, R. Bott, The Yang-Mills equations over Riemann surfaces, Philos. Trans. Roy. Soc. London Ser. A 308 (1983), 523-615.

[4] V. Baranovsky, V. Ginzburg, Conjugacy classes in loop groups and G-bundles on elliptic curves, Internat. Math. Res. Notices (1996), no. 15, 733-751.

[5] E. Brieskorn, Singular elements of semisimple algebraic groups, in: Actes du Congrès International des Mathématiciens (Nice, 1970), Tome 2, Gauthier-Villars, Paris, 1971, 279-284.

[6] G. Brüchert, Trace class elements and cross sections in Kac-Moody groups, Canad. J. Math. 50 (1998), 972-1006.

[7] P. Etingof, I. B. Frenkel, Central extensions of current groups in two dimensions, Comm. Math. Phys. 165 (1994), 429-444.

[8] I. B. Frenkel, Orbital theory for affine Lie algebras, Invent. Math. 77 (1984), 301-352.

[9] R. Friedman, J. Morgan, Holomorphic principal bundles over elliptic curves, Duke eprint math.AG/9811130.

[10] R. Friedman, J. Morgan, E. Witten, Vector bundles and F-theory, Comm. Math. Phys. 187 (1997), 679-743.

[11] S. Helmke, Loop groups and elliptic singularities, in: Proc. Kinosaki Conf. on Algebraic Geometry, Nov. 1998, 98-105.

[12] S. Helmke, P. Slodowy, Loop groups, principal bundles over elliptic curves and elliptic singularities, in: Annual Meeting of the Math. Soc. of Japan, Hiroshima, Sept. 1999, Abstracts, Section Infinite-Dimensional Analysis, 67-77.

[13] S. Helmke, P. Slodowy, On unstable principal bundles over elliptic curves, Publ. Res. Inst. Math. Sci. 37 (2001), 349-395.

[14] V. G. Kac, Constructing groups associated to infinite dimensional Lie algebras, in: InfiniteDimensional Groups with Applications (Berkeley, 1984), Math. Sci. Res. Inst. Publ. 4, Springer, New York, 1985, 167-216.

[15] V. G. Kac, Infinite Dimensional Lie Algebras, third edition, Cambridge University Press, 1990.

[16] E. J. N. Looijenga, Root systems and elliptic curves, Invent. Math. 38 (1976), 17-32.

[17] E. J. N. Looijenga, On the semi-universal deformation of a simple elliptic singularity II, Topology 17 (1978), 23-40.

[18] E. J. N. Looijenga, Invariant theory for generalized root systems, Invent. Math. 61 (1980), $1-32$.

[19] J. Y. Mérindol, Les singularités simples elliptiques, leurs déformations, les surfaces de Del Pezzo et les transformations quadratiques, Ann. Scient. École Norm. Sup. (4) 15 (1982), 17-44. 
[20] H. C. Pinkham, Simple elliptic singularities, Del Pezzo surfaces and Cremona transformations, Proc. Symp. Pure Math. 30 (1977), 69-71.

[21] A. Pressley, G. Segal, Loop Groups, Oxford University Press, New York, 1986.

[22] A. Ramanathan, Stable principal bundles on a compact Riemann surface, Math. Ann. 213 (1975), 129-152.

[23] K. Saito, Einfach elliptische Singularitäten, Invent. Math. 23 (1974), 289-325.

[24] K. Saito, Period mapping associated to a primitive form, Publ. Res. Inst. Math. Sci. 19 (1983), 1231-1264.

[25] K. Saito, Extended affine root systems I. Coxeter transformations, Publ. Res. Inst. Math. Sci. 21 (1985), 75-179.

[26] K. Saito, Extended affine root systems II. Flat invariants, Publ. Res. Inst. Math. Sci. 26 (1990), 15-78.

[27] G. Segal, Unitary representations of some infinite-dimensional groups, Comm. Math. Phys. 80 (1981), 301-342.

[28] P. Slodowy, Simple Singularities and Simple Algebraic Groups, Lecture Notes in Math. 815, Springer, Berlin, 1980.

[29] P. Slodowy, Chevalley groups over $C((t))$ and deformations of simply elliptic singularities, Sūrikaisekikenkyūsho Kōkyūroku 415 (1981), 19-38; also in: Algebraic Geometry (La Rábida, 1981), Lecture Notes in Math. 961, Springer, Berlin, 1982, 285-301.

[30] P. Slodowy, Singularitäten, Kac-Moody Liealgebren, assoziierte Gruppen und Verallgemeinerungen, Habilitationsschrift, Universität Bonn, 1984.

[31] P. Slodowy, A character approach to Looijenga's invariant theory for generalized root systems, Compositio Math. 55 (1985), 3-32.

[32] P. Slodowy, An adjoint quotient for certain groups attached to Kac-Moody algebras, in: Infinite-Dimensional Groups with Applications (Berkeley, 1984), Math. Sci. Res. Inst. Publ. 4, Springer, New York, 1985, 307-333.

[33] P. Slodowy, On the algebraic geometry of Kac-Moody groups, Sūrikaisekikenkyūsho Kōkyūroku 1086 (1999), 71-87.

[34] H. Yamada, Lie group theoretical construction of period mapping, Math. Z. 220 (1995), 231-255. 